\title{
IMPLEMENTATION OF DIGITAL IMAGE WATERMARKING TECHNIQUES USING DWT AND DWT_SVD METHOD
}

\author{
Smita Patil ${ }^{1}$, P. V. Baviskar ${ }^{2}$ \\ ${ }^{I}$ Department of Electronics \& Telecommunication, SSVPS's, B.S.D., College of Engineering, Dhule-424005, \\ Maharashtra, India \\ ${ }^{2}$ Department of Electronics \& Telecommunication, SSVPS's, B.S.D., College of Engineering, Dhule-424005, \\ Maharashtra, India
}

\begin{abstract}
These days, in every field there is gigantic utilization of computerized substance. Data took care of on web and mixed media system framework is in advanced structure. Computerized watermarking is only the innovation in which there is inserting of different data in advanced substance, which we need to shield from illicit replicating. Computerized picture watermarking is concealing data in any structure (content, picture, sound and video) in unique picture without corrupting its perceptual quality. On the off chance that of Discrete Wavelet Transform (DWT), deterioration of the first picture is completed to insert the watermark. Moreover, if there should arise an occurrence of cross breed system (DWT-SVD) firstly picture is decayed by and after that watermark is installed in solitary qualities acquired by application of Singular Value Decomposition (SVD). DWT and SVD are utilized in combination to enhance the nature of watermarking. We have the procedures which are looked at on the premise of Peak Signal to Noise Ratio (PSNR) esteem at various benefits of scaling component; high estimation of PSNR is coveted because it displays great intangibility of the strategy.
\end{abstract}

Keywords: PSNR, MSE and Hybrid DWT-SVD

\section{INTRODUCTION}

Since last couple of years, there is a noteworthy issue around unapproved and illicit get to and control of media records over web. Almost everyone can get copies of copyrighted sight and sound straight forwardly. So we need to make a lively system remembering the deciding objective to secure the copy benefits of media. Automated watermarking gives copyright security of data. The Watermarking is a methodology wherein the main picture generally called spread picture is changed by watermark picture. Certain properties of the spread picture are changed remembering the finished objective to disguise the data used for the recognizing verification of the proprietor of the principal substance [5].

Generally, watermarking uses two types of methods. Starting one is spatial space system another is change zone technique. In case of spatial space, the puzzle messages are generally embedded in the pixels of photo. The most generally perceived systems are histogram-based and LSB techniques in the spatial space. Watermarking frameworks which are based on spatial range, are sometimes supported over change space based watermarking systems in light of the way that the watermark set by them could be simply decimated and improved by the aggressors. However in the change space the watermark is embedded by altering the significance of coefficients in a change range with the assistance of discrete cosine change, discrete wavelet transform (DWT), discrete Fourier change and specific quality disintegration (SVD) methods.

\subsection{General Watermarking Methods}

In digital watermark, the information is generally embedded in a single, sometimes, the difference between the signal of watermarked and the signal of cover also refers as digital watermark. In the host signal, watermark has to be embedded. Embedding, attack, and detection are three important distinct steps in watermarking. In case of embedding, an algorithm accepts the host and subsequently it embeds the data, which ultimately resulted into the watermarked signal. In the next step, the watermarked digital signal proceeds to transmission and finally it gets stored, usually it gets transmitted to another person. If the modifications happen at this persons end, this is termedas attack. Though these modifications are not malicious, the term attack ascends from application of copyright protection, where third parties may challenge to eliminate the digital watermark by doing some modifications. Many potential modifications could happen, for example, lossy compression of the data (diminishing of resolution), cutting an image or video or purposefully additional of some noise. Detection (i.e. extraction) is an examples of algorithm generally used to attacked the signal to identify and remove the watermark from it. If the signal remains unmodified during the process of transmission, in this case watermark could be extracted. The digital watermarking applications 
are robust in nature and the process of algorithm extraction has to yield the watermark correctly, under strong modifications scenario. If changes made to the signal, then the fragile digital watermarking is unsuccessful.

\section{LITERATURE SURVEY}

Various studies have proposed various procedures of composing For example, Hui-Yu Huang [1] proposed system lossless data disguising procedure for a DWT. Our proposed strategy uses quantization segments for DWT, which could offer higher covering point of confinement which could protect the photo way of stego-pictures. In order to recover the principal picture losslessly, the riddle data has to be removed from stego-images. Cui-ling JIANG et al., [2] have offered the spread picture which is detached into non-covering squares of $16 \times 16$ pixels instead of standard parceling spread picture into $8 \times 8$ pieces and the DCT is used to change each square. The beauty of the DCT coefficients is, they are quantized and subsequently embedded the messages secretly. However, this strategy has many Steganography limitations which favored stegopicture quality over interchange procedures. The lossless data covering methodology for a DWT-based technique has recently been proposed by Hui-Yu Huang et al., [5].In this paper we proposed our strategy which uses quantization segments for DWT, this approach could offer higher covering utmost and recovery the photo way of stegopictures. By using our approach, the primary picture could be well again losslessly even when the puzzle data had been barred from stego-pictures. Satyanarayana Murty et al. [10] suggested procedure (DWT DCT-SVD) is exceptionally solid and can restrict various picture planning ambushes. The way of the watermarked picture is awesome to the extent recognizable quality and PSNR (42db). The proposed count is given off an impression of being lively to regardless of the ambushes said before from JPEG 2000. The singular qualities in every quadrant are later balanced by the specific estimations of the DWT-DCT changed visual watermark. We have demonstrated that by introducing the data at minimum frequencies is impenetrable to most of the strikes and ambushes at some extent are impenetrable to other repeat bunches. Moreover, Vishal Verma et al.., [11] have also successfully demonstrated an unequivocal survey of existing picture watermarking methodologies. In this paper, we describe the frameworks in perspective of different territories in which data is embedded. We have also examined particular sort of frameworks of introducing watermark and as indicated by come to fruition showed our proposed system in light of SVD DWT cream technique which have higher PSNR for extracted watermark picture. In order to further improve the watermarking in cutting edge picture, watermark could be embedded by using new lifted wavelets and also stationary wavelets, S-change etc. This approach would certainly further improve the watermarking technique.

\section{PROPOSED SYSTEM}

We have proposed the watermarking technique which is shown below. This method aims to embed a secret message or log into DWT coefficient at medium to high frequency parts and which subsequently bring back the original image coefficient when the secret message gets retrieved. The wavelet transform is very useful for conversion of a picture from spatial domain to frequency domain. In this paper we proposed the pairing of decomposition of digital picture to waveform with high frequency which generally corresponds to thorough parts of an image and low frequency corresponds to smooth parts of the image. The message which is digitally marked will be patched in high frequency parts and the image has to be rebuilt for cover image which will have concealed digital marked message. In this methodology, the embedded image gets decayed into inverse discrete wavelet transform (IDWT). The IDWT is generally very useful to transform from frequency domain to spatial domain. Therefore it's called as frequency-time representation. By using DWT method, patched image will get retrieved in to sub-band frequencies. We propose to take the digital marked data from the medium to high frequency parts and the retrieved digital marked data is going to perform matching through original message which includes the process of embedding and extraction:

\subsection{Algorithm}

\subsubsection{DWT-Embedding Algorithm}

1. Read \& resize the cover image $(512 \times 512)$

2 . Read \& resize the watermark logo $(512 \times 512)$

3. Decomposed cover image to first stage decomposition.

4. Application of dwt method on message image.

5. Entering of scale factor.

6. Modification ofhh sub band and its component.

(hh_mod $=\mathrm{hh}+\mathrm{f} * \mathrm{wl}$ hh $)$

7. Application ofidwt method with altered hh sub band.

8. Obtaining of watermarked image.

9. Addition of noise on watermarked image.

\subsubsection{Extraction of Algorithm of DWT}

1. Application of dwt method on watermarked image.

2. Identify the difference use below equation.

hh_Ext $=\left(\mathrm{hh} \_\right.$wl $\left.-\mathrm{hh}\right) / \mathrm{f}$

3. Application of idwt-2 using retrieved hh sub band component.

4. Obtaining the retrieved message and original image.

5. Calculation of PSNR, NC and MSE.

\subsubsection{DWT_SVD: Embedding Algorithm}

1.Read and resize the cover image $(512 \times 512)$

2. Read and resize the watermark logo $(256 \times 256)$

3. Application of haar wavelet method and decompose cover image into further $4 \mathrm{sub}$ bands.

4. Application of SVD method on hh sub band: (U hh, S_hh, V_hh

5. Application of SVD method on watermark logo.

6. Entering scale-factor (f)

7. Modification of S component, from svd method, of hh band

8. S hh $\bmod =\mathrm{S} h \mathrm{hh}+\mathrm{f} * \mathrm{~S}$ wm;

9. Application ISV $\bar{D}$ method on modified hh part. 
10. Application idwt method with modified hh band for obtaining watermarked image.

11. Addition of noise onto watermarked image.

\subsubsection{Extracting Process Algorithm of DWT_SVD}

1. Application of haar wavelet method to watermarked image.

2. Application of SVD method to HH band.

3. $\mathrm{D}=\left(\mathrm{S} \_w m I-\mathrm{S} \_h h\right) / \mathrm{f}$.

4. Extraction of S parts of watermark logo.

5. Application inverse-SVD method.

6. Obtaining watermark logo.

7. Calculation of PSNR, MSE \& NC

The steps of evaluation and comparison of the performance of DWT and DWT-SVD are given by

$$
\text { MSE }=\sum_{i=0}^{M-1} \frac{\left[I(i, j)-I^{\prime}(i, j)\right.}{M \times N}
$$

Where,

MSE is mean square error,

$\mathrm{M} \times \mathrm{N}$ is the dimension of images,

$\mathrm{I}(\mathrm{i}, \mathrm{j})$ is original image dimension,

I' $(i, j)$ is the dimension of watermarked image

By using the above result of MSE PSNR can be calculated using the formula,

$$
\text { PSNR }=10 \log _{10}\left(\frac{255^{2}}{\mathrm{MSE}}\right)
$$

For evaluation of similarities between original cover image and obtained watermarks the Normalize correlation coefficient are given by,

$$
\mathrm{NC}=\frac{\sum_{i=1}^{N} W i W i^{\prime}}{\sqrt{\sum_{i=1}^{N} W t} \sqrt{\sum_{i=1}^{N} W t^{\prime}}}
$$

\subsection{Mathematical Model}

\section{S $\{$ I,CI,D,EM,ID,EI,DE,EHH,OM $\}$}

$\mathrm{I}=\mathrm{I}$ is set of input image.

$\mathrm{I}=\{\mathrm{I} 1, \mathrm{I} 2 \ldots \mathrm{In}\}$

$\mathrm{CI}=\mathrm{CI}$ is set of cover image.

$\mathrm{CI}=\{\mathrm{CI} 1, \mathrm{CI} 2 \ldots \mathrm{CIn}\}$

$\mathrm{D}=\mathrm{D}$ is set of DWT method.

$\mathrm{D}=\{\mathrm{D} 1, \mathrm{D} 2, \ldots . \mathrm{Dn}\}$

$\mathrm{EM}=\mathrm{EM}$ is set of embedding message.

$\mathrm{EM}=\{\mathrm{EM} 1, \mathrm{EM} 2 \ldots \mathrm{EMn}\}$
ID $=$ ID is set of IDWT method

$\mathrm{ID}=\{\mathrm{ID} 1, \mathrm{ID} 2 \ldots . \mathrm{IDn}\}$

$\mathrm{EI}=\mathrm{EI}$ is set of embedding image.

$\mathrm{EI}=\{\mathrm{EI} 1, \mathrm{EI} 2 \ldots \mathrm{EIn}\}$

$\mathrm{DE}=\mathrm{DE}$ is set $\mathrm{DWT}$ for extraction

$\mathrm{DE}=\{\mathrm{DE} 1, \mathrm{DE} 2 \ldots . \mathrm{DE} n\}$

$\mathrm{EHH}=\mathrm{EHH}$ is set of extraction of $\mathrm{HH}$ frequency.

$\mathrm{EHH}=\{\mathrm{EHH} 1, \mathrm{EHH} 2 \ldots \mathrm{EHHn}\}$

$\mathrm{OM}=\mathrm{OM}$ is set of output images.

$\mathrm{OM}=\{\mathrm{OM} 1, \mathrm{OM} 2 \ldots \mathrm{OMn}\}$

\subsection{Process}

1) Take input image. $\mathrm{I}=\{\mathrm{I} 1, \mathrm{I} 2 \ldots \mathrm{In}\}$

2) Take cover and message image $\mathrm{CI}=\{\mathrm{CI} 1, \mathrm{CI} 2 \ldots \mathrm{CIn}\}$

3) Apply DWT method. $\mathrm{D}=\{\mathrm{D} 1, \mathrm{D} 2, \ldots . \mathrm{Dn}\}$

4) Embedding message image into cover image. $\mathrm{EM}=\{\mathrm{EM} 1, \mathrm{EM} 2 \ldots \mathrm{EMn}\}$

5) Apply IDWT. $\mathrm{ID}=\{\mathrm{ID} 1, \mathrm{ID} 2 \ldots . \mathrm{IDn}\}$

6) Take Embedding image for extraction. $\mathrm{EI}=\{\mathrm{EI} 1, \mathrm{EI} 2 \ldots \mathrm{EIn}\}$

7) Apply DWT for Extraction. $\mathrm{DE}=\{\mathrm{DE} 1, \mathrm{DE} 2 \ldots . \mathrm{DEn}\}$

8) Extract $\mathrm{HH}$ frequency component. $\mathrm{EHH}=\{\mathrm{EHH} 1, \mathrm{EHH} 2 \ldots \mathrm{EHHn}\}$

9) Original image. $\mathrm{OM}=\{\mathrm{OM} 1, \mathrm{OM} 2 \ldots \mathrm{OMn}\}$

Result: We get original image.

\subsection{System Architecture}

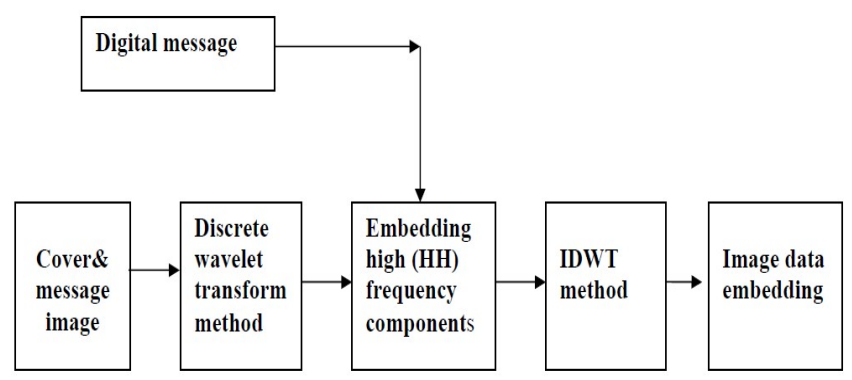

Fig1 (a) Embeding process

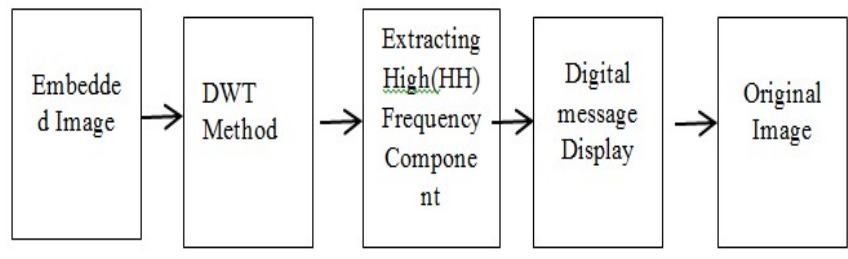

Fig1 (b) Extracting process 


\section{RESULT}

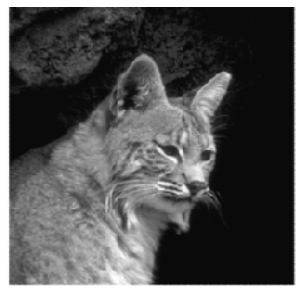

(a) Original Image

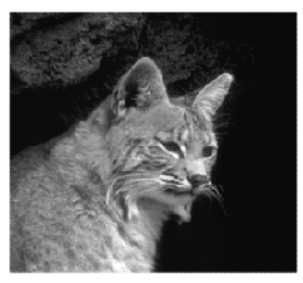

(a) Watermarked Image

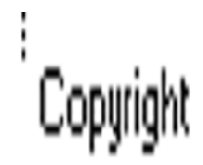

(b) Watermarked Logo

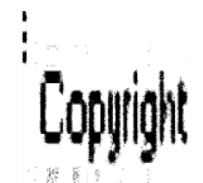

(b) Extracted Logo

Fig 3 Matlab Simulation result for single level DWT with Gauassian attack

The PSNR value of extracted logo in DWT SVD after extraction is observed to be 87.09 and 91.26 , respectively. These values indicate the accuracy of, DWT-SVD over DWT technique (Table 1). The scale factors were added for safety reasons for purses. We offered the method, in which the values of the scale factors are observed to be in continuous range from 0.01 to 0.07 (Table 1). Our results clearly indicated that, when the scale factor was larger, the robustness was managed efficiently. In contrast, quality of extracted logo was observed to be good when the scale factor was smaller. We also evaluated the robustness of the new watermarking scheme with several attacks which includes addition of Speckle noise, salt \& pepper noise, Gaussian low pass filter and even Local variance noise. Table 2 represents, the MSE and PSNR of distorted watermarked images under above distortions. The PSNR was observed to be less in comparison with hybrid technique (DWT-SVD), when the watermarked image using DWT technique was attacked.

Table 1: Comparison between PSNR (db) of others \& proposed method

\begin{tabular}{|l|l|l|l|l|}
\hline $\begin{array}{l}\text { Scaling } \\
\text { factor } \\
\text { values }\end{array}$ & $\begin{array}{l}\text { PSNR } \\
(\mathrm{db}) \text { for } \\
\text { others } \\
\text { method } \\
\text { DWT }\end{array}$ & $\begin{array}{l}\text { PSNR (db) } \\
\text { values for } \\
\text { DWT_SVD } \\
\text { (other } \\
\text { method) }\end{array}$ & $\begin{array}{l}\text { PSNR (db) } \\
\text { values for } \\
\text { DWT } \\
\text { (Proposed } \\
\text { Method) } \\
\text { after } \\
\text { extraction }\end{array}$ & $\begin{array}{l}\text { PSNR (db) } \\
\text { values for } \\
\text { DWT-SVD } \\
\text { (Proposed } \\
\text { method) } \\
\text { after } \\
\text { extraction }\end{array}$ \\
\hline 0.01 & 43.63 & 76.2096 & 79.02 & 84.53 \\
\hline 0.02 & 37.58 & 60.8586 & 78.56 & 85.78 \\
\hline 0.03 & 34.08 & 52.6066 & 87.09 & 91.26 \\
\hline 0.04 & 31.57 & 49.4157 & 78.52 & 81.47 \\
\hline 0.05 & 29.64 & 45.9157 & 79.02 & 80.21 \\
\hline 0.06 & 28.04 & 43.1116 & 78.62 & 80.72 \\
\hline 0.07 & 26.71 & 40.8654 & 79.02 & 81.46 \\
\hline
\end{tabular}

Table2. Comparison of PSNR \& MSE of watermarked image against different attack byour proposed method

\begin{tabular}{|l|l|l|l|l|}
\hline $\begin{array}{l}\text { Cover } \\
\text { image }\end{array}$ & Attack & $\begin{array}{l}\text { Control } \\
\text { parameters }\end{array}$ & DWT & DWT_SVD \\
\hline \multirow{4}{*}{ CARS } & Gaussian & PSNR(db) & 49.4867 & 80.5406 \\
\cline { 2 - 5 } & & MSE & 0.73184 & 0.00057414 \\
\cline { 2 - 5 } & Local variance & PSNR(db) & 48.2597 & 80.5463 \\
\cline { 2 - 5 } & & MSE & 0.38647 & 0.00057336 \\
\cline { 2 - 5 } & Speckle & PSNR(db) & 49.7416 & 79.8675 \\
\cline { 2 - 5 } & & MSE & 0.69011 & 0.0006704 \\
\cline { 2 - 5 } & Pepper \& salt & PSNR(db) & 60.96667 & 79.3662 \\
\cline { 2 - 5 } & & MSE & 0.05201 & 0.00075242 \\
\hline \multirow{5}{*}{ Couple } & Gaussian & PSNR(db) & 49.4419 & 80.721 \\
\cline { 2 - 5 } & & MSE & 0.73942 & 0.000550 \\
\cline { 2 - 5 } & Local variance & PSNR(db) & 52.2286 & 80.1298 \\
\cline { 2 - 5 } & & MSE & 0.3892 & 0.0005013 \\
\cline { 2 - 5 } & Speckle & PSNR(db) & 45.702 & 79.6314 \\
\cline { 2 - 5 } & & MSE & 1.7494 & 0.0007078 \\
\cline { 2 - 5 } & Pepper \& salt & PSNR(db) & 53.2131 & 79.5002 \\
\cline { 2 - 5 } & & MSE & 0.3103 & 0.0007295 \\
\hline
\end{tabular}

Table 3: Using DWT-SVD method, the PSNR (db), MSE \& $\mathrm{NC}$ of watermarked noisy image on different logo.

\begin{tabular}{|l|l|l|l|l|}
\hline $\begin{array}{l}\text { Gaussian } \\
\text { Noisy } \\
\text { Image }\end{array}$ & $\begin{array}{l}\text { Extracted } \\
\text { Logo }\end{array}$ & PSNR & MSE & NC \\
\hline & $\vdots$ & 66.1926 & 0.015625 & 0.99194 \\
\hline & Copulght & & & \\
\hline & Wold & & & \\
\hline
\end{tabular}

The results obtained by simulation are presented in Table 3 . The different images of Gaussian noisy, by using hybrid technique we embedded different logos and the subsequent simulation result are tabulated in table-3. The combination of DWT and SVD are used for improvement of quality of the watermarking fusion which resulted into the very striking technique.

\section{CONCLUSION}

On looking at the estimations of PSNR at various benefits of mounting component $\mathrm{C}$, it's presumed that the cross breed system DWT-SVD is vastly improved as compared to DWT procedure. We have tried DWT-SVD half breed strategy, the scale component diminishes as the PSNR increments. Lower the estimation of PSNR, higher would be the corruption in the nature at the first picture. Half breed 
method utilized in combination to enhance the nature of watermarking. The strategy proposed in this paper where the distinction from customary plan is that the insertion of watermarking in high recurrence segments which gives prodigious execution in an assortment of picture application.

\section{ACKNOWLEDGEMENT}

The authors would like to thank the Principal, Head of Department (Department of Electronics and Telecommunication), SSVPS, B. S. D., College of Engineering, Dhule (North Maharashtra University) for providing necessary facilities for carrying out the work. Authors also thankful to the anonymous reviewer for their valuable comments and further improvement of the manuscript.

\section{REFERENCES}

[1]. Hui-Yu Huang and Shih-Hsu Chang "A lossless data hiding based on discrete Haar wavelet transform", 10th IEEE International Conference on Computer and Information Technology, 2010.

[2]. Cui-ling JIANG “A Steganographic Method based on the JPEG Digital images" Institute of Information, East China University of Science and Technology, 2011.

[3]. Anjali A. Shejuland U.L Kulkarni "A DWT based Approach for Steganography Using Biometrics", International Conference on Data Storage and Data Engineering, 2010.

[4]. Souvik Bhattacharyya and Gautam Sanyal "Data Hiding in Images in Discrete Wavelet Domain Using PMM", World Academy of Science, Engineering and Technology, 2010.

[5]. Mohammad Reza Soheili "A Robust Digital image Watermarking Scheme Based on DWT" Journal of Advances in Computer Research, m2(2010) 75-82.1.

[6]. Qing zhong Li, Chen $\mathrm{Yu}$ and Dongsheng Chu "A Robust Image Hiding Method Based on Sign Embedding and Fuzzy Classification", Proceedings of the 6th World Congress on Intelligent Control and Automation, 2006.

[7]. Po-Yueh Chen and Hung-Ju Lin, "A DWT Based Approach for Image Steganography", IEEE International Journal of Applied Science and Engineering, 2006.

[8]. Guorong Xuan, Yun Q. Shi and Chengyun Yang "Lossless Data Hiding Using Integer Wavelet Transform and Threshold Embedding Technique" 0-7803-93325/05/\$20.00 C2005 IEEE.

[9]. Y. K. Lee and L.-H. Chen, "High capacity image steganographic model", Vision, Image and Signal Processing, IEEE Proceedings, 2000.

[10]. Satyanarayana Murty and P. Rajesh Kumar, "A Robust Digital Image Watermarking Scheme Using Hybrid DWTDCT-SVD Technique" IJCSNS International Journal of Computer Science and Network Security, VOL.10 No.10, October 2010.

[11]. Vishal Verma and Jyotsna Singh "Digital Image Watermarking Techniques: A Comparative Study" International Journal of Advances in Electrical and Electronics Engineering ISSN: 2319-1112 /V2N1:173$184,2013$.
[12]. Nidhi Bisla and Prachi Chaudhary "Comparative Study of DWT and DWT-SVD Image Watermarking Techniques" International Journal of Advanced Research in Computer Science and Software Engineering Volume 3, Issue6, June 2013 ISSN: 2277 128X

\section{BIOGRAPHIES}

Ms. Smita Patil has completed her B.E. (Electronics and Telecommunication) from the Pune University, Pune. She has been pursuing M.E. (Communication Systems) from SSVPS's, B.S.D., College of Engineering, Dhule (North Maharashtra University, Jalgaon)

Associate Prof. P. V. Baviskar has more than 26 years of experience in the field of Electronics. He has published paper on "Nanoscale SOI MOSFET structures and study of performance factors", IJCA. 